\title{
Leveraging electronic health record data to improve sepsis surveillance
}

\author{
Claire N Shappell, ${ }^{1,2}$ Chanu Rhee ${ }^{1,3}$
}

${ }^{1}$ Department of Population Medicine, Harvard Medical School/Harvard Pilgrim Health Care Institute, Boston, Massachusetts, USA

${ }^{2}$ Division of Pulmonary and Critical Care Medicine, Brigham and Women's Hospital, Boston, Massachusetts, USA

${ }^{3}$ Division of Infectious Diseases, Brigham and Women's Hospital, Boston, Massachusetts, USA

\section{Correspondence to} Dr Chanu Rhee, Department of Population Medicine, Harvard Medical School/Harvard Pilgrim Health Care Institute, Boston, MA 02215, USA;

crhee@bwh.harvard.edu

Accepted 18 February 2020 Published Online First

27 February 2020

\section{SLinked}

- http://dx.doi.org/10.1136/ bmjqs-2019-010123

\section{Check for updates}

(c) Author(s) (or their employer(s)) 2020. No commercial re-use. See rights and permissions. Published by BMJ.

To cite: Shappell CN, Rhee C. BMJ Qual Saf

2020;29:706-710.
Sepsis, the syndrome of life-threatening organ dysfunction that complicates severe infection, is a leading cause of death and disability worldwide. ${ }^{1}$ A growing recognition of the enormous burden of sepsis has spurred numerous awareness campaigns, quality improvement initiatives and regulatory measures in recent years. Reliably tracking the burden of sepsis is challenging, however, because sepsis is a clinical syndrome based on a constellation of non-specific signs and symptoms and lacks a gold standard for diagnosis. ${ }^{2}$ Given the substantial resources being dedicated to improving sepsis care and outcomes, a parallel investment in developing robust, high-quality surveillance tools is necessary to understand which initiatives are effective and where best to allocate future resources.

Until recently, sepsis surveillance has primarily been conducted using hospital discharge diagnosis codes. Epidemiological studies using these data have consistently shown dramatic increases in sepsis incidence and declines in case fatality rates over the past several decades. ${ }^{3-5}$ However, this method is seriously flawed since it requires (1) clinicians to recognise sepsis by identifying that infection is present and responsible for organ dysfunction; (2) clinicians to document sepsis in the medical record and (3) hospital coders to appropriately identify this documentation and assign sepsis as a primary or secondary diagnosis. These steps are subjective and easily biassed by changing diagnosis and coding practices over time. Specifically, education and awareness campaigns, new screening protocols and international guidelines are all constantly encouraging early detection of sepsis and organ dysfunction. This, by design, leads to the diagnosis of 'sepsis' in more mildly ill patients that previously might only have been labelled by their specific infection (eg, pneumonia) or non-specific illnesses. ${ }^{6-9}$ In the USA, where sepsis diagnoses are tied to the highest level of patient complexity and reimbursement, hospitals also have a clear financial incentive to code for sepsis. ${ }^{10}$ Diagnosing earlier and milder forms of sepsis may benefit patients, but it creates an ascertainment bias for surveillance since it is difficult to know whether the reported increases in sepsis incidence and declining mortality rates reflect true changes in disease epidemiology and better sepsis care, or simply artefacts from the inclusion of more patients with less severe illness in the denominator. ${ }^{11}$

Some healthcare systems have used prospective registries based on various screening protocols to track sepsis outcomes. $^{12} 13$ However, this method is also vulnerable to ascertainment bias since the implementation of these screens tends to enhance early identification of sepsis and therefore also captures increasingly milder forms of sepsis. Prospective registries are also resource-intensive and have limited comparability across hospitals and geographical regions due to heterogeneous inclusion criteria. Death records are another data source that have been used to generate national and global estimates of sepsis mortality, but physicians are notoriously inaccurate at coding causes of death and sepsis in particular tends to be under-coded. ${ }^{114}$ Furthermore, trends in the coding of sepsis on death certificates are subject to the same changes in diagnosis and documentation practices as hospital administrative data. ${ }^{15}$

The need for a more objective, consistent and scalable approach to sepsis surveillance has recently led some researchers and policymakers to turn to direct clinical indicators of sepsis that can be extracted from electronic health record (EHR) systems which are increasingly ubiquitous in the USA and other developed countries. ${ }^{16}$ A prominent example of this 
approach is the 'Adult Sepsis Event' (ASE) definition created by the US Centers for Disease Control and Prevention in $2018 .{ }^{17}$ The ASE was conceptually based on the Third International Consensus Definitions for Sepsis and Septic Shock (Sepsis-3) framework of sepsis as infection with concurrent organ dysfunction, ${ }^{18}$ but was optimised for retrospective surveillance across a broad range of hospitals using data routinely available in EHRs rather than for real-time decision-making. The ASE identifies hospitalisations with presumed serious infection, as defined by a blood culture order and administration of at least 4 days or antibiotics (or fewer in cases of death, discharge to hospice, transfer to another acute care hospital, or transition to comfort measures before 4 days), and concurrent acute organ dysfunction, defined as initiation of vasopressors or mechanical ventilation, elevated lactate, or clearly defined changes in creatinine, total bilirubin or platelets from patients' baseline values. ASE requires four antibiotic days to improve specificity by excluding patients who only briefly receive empiric antibiotics and also mitigate potential bias from increased screening and decreasing thresholds to start empiric antibiotics for suspected sepsis. The ASE organ dysfunction criteria resemble the Sequential Organ Failure Assessment (SOFA) score used by Sepsis- $3,{ }^{18}$ but use binary thresholds and a smaller number of data elements for greater simplicity to enable use in a wide range of hospitals and EHR systems (table 1).

The ASE definition was initially developed as part of a 2017 multicenter study of the burden of sepsis in the USA and applied across a nationally representative cohort of 409 diverse hospitals from seven datasets. ${ }^{19}$ This study yielded a sepsis prevalence rate of $6 \%$ in hospitalised adult patients and an in-hospital mortality rate of $15 \%$; when extrapolated nationwide, this generated an estimated 1.7 million adult sepsis cases and 270000 associated deaths. On medical record reviews, ASE criteria had reasonable sensitivity (69.7\%) and good specificity (98.1\%) compared with the clinical Sepsis-3 definition. Many of the false positives and false negatives, however, were due to intentional mismatches between the ASE organ dysfunction criteria and the SOFA score used by the Sepsis-3 definition, as the ASE criteria were designed to simplify the number of data elements to facilitate consistent implementation across different EHR systems (eg, by identifying respiratory failure by mechanical ventilation alone rather than $\mathrm{PaO} 2 / \mathrm{FiO} 2$ ratios, using any vasopressor initiation rather than specific vasopressor doses, and excluding Glasgow Coma Scale scores). Therefore, the 'accuracy' of ASE depends on whether one truly considers Sepsis-3 to be the 'gold standard' for sepsis diagnosis. When used to examine sepsis incidence and mortality from 2009 to 2014, the ASE definition generated much more stable trends compared with administrative definitions, and in fact no significant change in incidence or combined death or discharge to hospice was seen when the lactate criteria was omitted (an a priori decision due to increased lactate testing over the period studied).

The ASE was the beginning of an important paradigm shift towards population-level sepsis surveillance using EHR data, but it is certainly not the end. In this issue of BMJ Quality \& Safety, Valik and colleagues present the first validation of an EHR-based algorithm based directly on Sepsis-3 criteria and its application to measure sepsis incidence, mortality and variation across non-ICU wards in a Swedish academic medical centre. ${ }^{20}$ As per the work by Seymour and the Sepsis-3 task force, ${ }^{21}$ suspected infection was defined as any culture obtained (not just blood cultures) and at least two doses of antimicrobials administered, while organ dysfunction was defined by a rise in maximum SOFA score around the time of infection onset by at least two points compared with a baseline SOFA score (table 1). On medical record review, this algorithm achieved very high sensitivity $(88.7 \%)$, specificity (98.5\%) and positive predictive value (PPV) (88.1\%) relative to Sepsis-3 criteria as determined by two infectious disease physicians. The performance was excellent across both community-onset and hospital-onset sepsis-an important finding given that administrative data can only distinguish these two conditions by present-on-admission codes, which are often inaccurate and variably applied across hospitals. ${ }^{22}$ Sensitivity analyses using alternative definitions of suspected infection, including blood cultures and 4 days of antibiotics as in the ASE definition, had lower sensitivity (71.8\% for the ASE equivalent) though improved specificity and PPV (99.2\% and 91.7\%, respectively). When the algorithm was applied to the hospital's population over a 1.5 -year period, it identified $10.4 \%$ of patients as septic (1.3\% hospital-onset and 9.1\% community-onset sepsis), with an in-hospital mortality rate of $8.6 \%$.

This study provides further evidence that EHR data can be used to build an accurate automated sepsis surveillance system, and is the first medical recordbased validation of an algorithm based directly on the SOFA score and Sepsis-3 criteria. The mortality rate of $8.6 \%$ is substantially lower than ASE's mortality rate but is close to the $10 \%$ rate in US cohorts used for the derivation and validation of Sepsis-3 criteria, ${ }^{21}$ suggesting at least some degree of generalisability. As the authors assert, the Sepsis-3 algorithm identifies a less severely ill set of patients than ASE and therefore may be more relevant for surveillance of general (nonICU) wards. ${ }^{23}$ Furthermore, while the requirement for only two doses of antimicrobials in their definition of suspected infection may cost some specificity, it allows for the possibility of prospective monitoring of sepsis cases as they develop in the hospital and influencing real-time clinical decision-making to improve sepsis care. 
Table 1 Comparison of automated Sepsis-3 algorithm and CDC adult sepsis event criteria

\begin{tabular}{|c|c|c|}
\hline Criteria & Sepsis-3 algorithm as implemented by Valik et al & CDC Adult Sepsis Event \\
\hline Infection & $\begin{array}{l}\text { 1. Any clinical culture obtained, AND } \\
\text { 2. } \geq 2 \text { antibiotic doses within } 6-48 \text { hours } \\
\text { Culture sites include abdomen, blood, bone, bronchoalveolar lavage, cerebral spinal } \\
\text { fluid, catheters/devices, nasopharynx, pleural space, skin/tissue, sputum, stool, } \\
\text { synovial fluid, urine. Cultures types include bacterial or C. difficile toxin, Mycoplasma } \\
\text { pneumoniae DNA, enterohemorrhagic E. coli DNA, Legionella urine antigen, fungal } \\
\text { cultures from blood. If antibiotic administration occurred first, a culture must be } \\
\text { obtained within } 24 \text { hours. If a culture was obtained first, an antibiotic must be given } \\
\text { within } 72 \text { hours. One antibiotic dose permitted if patient was admitted to the ICU } \\
\text { prior to } 24 \text { hours, or died prior to } 48 \text { hours from the first antibiotic dose. 'Onset of } \\
\text { infection' defined as the first of either event }\end{array}$ & $\begin{array}{l}\text { 1. Blood culture obtained, AND } \\
\text { 2. } \geq 4 \text { consecutive antibiotic days } \\
\text { Antibiotic sequence starts with a 'new' antibiotic (ie, not } \\
\text { given in prior } 2 \text { days) administered within } \pm 2 \text {-day window } \\
\text { around blood culture day. }<4 \text { antibiotic days permitted } \\
\text { if patient died, was discharged to hospice or another } \\
\text { hospital or transitioned to comfort measures before } 4 \\
\text { days. At least one antibiotic must be parenteral. 'Day of } \\
\text { infection onset' defined as the day of blood culture or first } \\
\text { antibiotic, whichever is earlier }\end{array}$ \\
\hline Organ Dysfunction & $\begin{array}{l}\text { Increase in modified SOFA Score by } \geq 2 \text { points from baseline during } \\
\text { window of up to } 48 \text { hours before to } 24 \text { hours after onset of infection: }\end{array}$ & $\begin{array}{l}\geq 1 \text { of the following "eSOFA" criteria within }+/-2 \\
\text { calendar days of blood culture day: }\end{array}$ \\
\hline Carc & $\begin{array}{l}1 \text { - Mean arterial pressure }<70 \mathrm{~mm} \mathrm{Hg} \\
\text { Baseline=last measured mean arterial pressure before suspected infection window } \\
\text { (only during current hospitalisation). Vasopressor doses not used since surveillance } \\
\text { performed outside the ICU }\end{array}$ & $\begin{array}{l}\text { Vasopressor initiation } \\
\text { Specific vasopressor must not have been given in prior } \\
\text { calendar day. Vasopressors given as bolus or in operating } \\
\text { room excluded }\end{array}$ \\
\hline Pulmonary & $\begin{array}{l}1-\mathrm{PaO} 2 / \mathrm{FiO} 2<400 \text { or } \mathrm{SpO} 2 / \mathrm{FiO} 2<512 \\
2-\mathrm{PaO} 2 / \mathrm{FiO} 2<300 \text { or } \mathrm{SpO} / \mathrm{FiO} 2<357 \\
3-\mathrm{PaO} 2 / \mathrm{FiO} 2<200 \text { or } \mathrm{SpO} 2 / \mathrm{FiO} 2<214\end{array}$ & $\begin{array}{l}\text { Mechanical ventilation initiation } \\
>1 \text { calendar day between ventilation episodes required }\end{array}$ \\
\hline
\end{tabular}

$3-\mathrm{PaO} 2 / \mathrm{FiO} 2<200$, or $\mathrm{Sp02} / \mathrm{FiO} 2<214$

$4-\mathrm{PaO} 2 / \mathrm{FiO} 2<100$, or Sp02/Fi02 $<89$

Baseline $=$ last $\mathrm{PaO} 2$ or SpO2 prior to suspected infection window during last 3 months. ICD codes for home oxygen or ventilator use in prior year=2 baseline points

\begin{tabular}{|c|c|c|}
\hline Renal & $\begin{array}{l}1 \text { - Creatinine } 110-170 \mu \mathrm{mol} / \mathrm{L} \\
2 \text { - Creatinine } 171-299 \mu \mathrm{mol} / \mathrm{L} \\
3 \text { - Creatinine } 300-440 \mu \mathrm{mol} / \mathrm{L} \\
4 \text { - Creatinine }>440 \mu \mathrm{mol} / \mathrm{L} \\
\text { Baseline=last measured creatinine prior to suspected infection window during last } \\
3 \text { months. ICD codes for chronic dialysis=4 baseline points. Urine output not used } \\
\text { due to data availability }\end{array}$ & $\begin{array}{l}\uparrow 2 x \text { Creatinine or } \downarrow \geq 50 \% \text { of estimated glomerular } \\
\text { filtration rate relative to baseline } \\
\text { Baseline creatinine=lowest during hospitalisation if } \\
\text { infection onset on hospital day } \leq 2 \text {, or lowest during } \pm 2 \text { - } \\
\text { day window period if infection onset on hospital day }>2 \text {. } \\
\text { Patients with ICD codes for end-stage renal disease } \\
\text { excluded }\end{array}$ \\
\hline Hepatic & $\begin{array}{l}1 \text { - Bilirubin } 20-32 \mu \mathrm{mol} / \mathrm{L} \\
2 \text { - Bilirubin } 33-101 \mu \mathrm{mol} / \mathrm{L} \\
3 \text { - Bilirubin } 102-204 \mu \mathrm{mol} / \mathrm{L} \\
4 \text { - Bilirubin }>204 \mu \mathrm{mol} / \mathrm{L} \\
\text { Baseline=last measured bilirubin prior to suspected infection window during last } \\
3 \text { months }\end{array}$ & $\begin{array}{l}\text { Bilirubin } \geq 2.0 \mathrm{mg} / \mathrm{dL} \text { and } \uparrow 2 \mathbf{x} \text { from baseline } \\
\text { Baseline bilirubin=lowest during hospitalisation if } \\
\text { infection onset on hospital day } \leq 2 \text {, or lowest during } \pm 2 \text { - } \\
\text { day window period if infection onset on hospital day }>2\end{array}$ \\
\hline Coagulation & $\begin{array}{l}1 \text { - Platelets } 100-149 \times 10^{3} / \mu \mathrm{L} \\
2 \text { - Platelets } 50-99 \times 10^{3} / \mu \mathrm{L} \\
3 \text { - Platelets } 20-49 \times 10^{3} / \mu \mathrm{L} \\
4 \text { - Platelets }<20 \times 10^{3} / \mu \mathrm{L} \\
\text { Baseline=last measured platelet count prior to suspected infection window during } \\
\text { last } 3 \text { months }\end{array}$ & $\begin{array}{l}\text { Platelet count }<100 \times 10^{3} / \mu \mathrm{L} \text { and } \downarrow \geq 50 \% \text { decline } \\
\text { from baseline (baseline must be } \geq 100 \text { ) } \\
\text { Baseline platelets=lowest during hospitalisation if } \\
\text { infection onset on hospital day } \leq 2 \text {, or lowest during } \pm 2 \text { - } \\
\text { day window period if infection onset on hospital day }>2\end{array}$ \\
\hline $\begin{array}{l}\text { Neurologic } \\
\text { (SOFA) or } \\
\text { Perfusion } \\
\text { (eSOFA) }\end{array}$ & $\begin{array}{l}1 \text { - Glasgow Coma Scale score } 13-14 \\
2 \text { - Glasgow Coma Scale score } 10-12 \\
3 \text { - Glasgow Coma Scale score 6-9 } \\
4 \text { - Glasgow Coma Scale score<6 } \\
\text { Baseline=last measured value before suspected window (only during current } \\
\text { hospitalisation). If Glasgow Coma Scale unavailable, structured data on 'alert' (0 } \\
\text { points) or 'not alert' (one point) used }\end{array}$ & Lactate $\geq 2.0 \mathrm{mmol} / \mathrm{L}$ \\
\hline
\end{tabular}

CDC, Centers for Disease Control and Prevention; FiO2, fraction of inspired oxygen; PaO2, arterial PaO2 of oxygen; SOFA, Sequential Organ Failure Assessment; SpO2, peripheral capillary oxygen saturation.

Despite these promising results, there are some caveats to this study that are worth noting. First, the algorithm was studied and validated in a single-centre population with a much lower burden of comorbid conditions compared with the multi-centre cohort in which the ASE was studied; it is therefore unclear whether the Sepsis-3 algorithm would maintain its high specificity if applied to a more medically complex population with a greater prevalence of pre-existing organ dysfunction. Second, ICU time was censored due to a lack of data on vital signs and medications, and so their estimations of sepsis incidence should interpreted with caution. Third, the extent to which the algorithm is susceptible to ascertainment bias from changing clinical practice over time (and changing data availability in EHRs) is unknown since the authors did not use it to track sepsis trends in their hospital.

More broadly, it is important to consider where the automated Sepsis-3 algorithm fits in the framework of sepsis definitions. Given the complexity of sepsis, 
no one set of criteria can suit the needs of all stakeholders. ${ }^{24}$ For example, clinicians require a definition optimised for sensitivity and ease of application at the bedside to facilitate timely treatment and avoid missing cases. In contrast, a surveillance definition is meant to reliably track sepsis over time and across different settings to characterise changes in disease epidemiology, interpret the impact of prevention and treatment initiatives, benchmark incidence and outcomes across facilities and geographical regions (and thus identify opportunities for improvement), and guide resource and research investments. As such, surveillance definitions typically prioritise specificity, objectivity and reproducibility over timely diagnosis. This sometimes means that ambiguous or mild cases are excluded. Furthermore, a low burden of measurement is important to facilitate widespread implementation.

With those considerations in mind, the automated Sepsis-3 algorithm appears to be very well-suited to track sepsis incidence and outcomes within the hospital where it was developed. However, it is unclear the degree to which consistent implementation of this approach across a diverse range of hospitals is feasible given the relative complexity of Sepsis-3 criteria and wide variability in the sophistication of EHR systems and data repositories. Prior work has demonstrated how seemingly minor variations in the definition and measurement of the traditional systemic inflammatory response syndrome-based sepsis criteria can have a major impact on the apparent incidence of sepsis. ${ }^{25}$ For the Sepsis-3 algorithm, identifying all potential clinical cultures as opposed to blood cultures alone (as per ASE criteria) dramatically expands the number of data elements that need to be identified and mapped. Furthermore, the SOFA score is highly sensitive to missing data and includes several elements that are inconsistently measured across hospitals and variably stored in EHRs, such as Glasgow Coma Scores, vasopressor doses, urine output and blood gas data. Indeed, even in this study, missing SOFA score data elements were common (particularly Glasgow Coma Scale and bilirubin), and several modifications of the SOFA score were needed based on data availability, such as use of peripheral capillary oxygen saturation instead of the $\mathrm{PaO} 2$ of oxygen and the omission of urine output. While these are relatively minor adaptations, they underscore the likelihood that slight variations in SOFA implementation are likely to occur across hospitals based on data availability, each of which could confound attempts at comparing sepsis rates and outcomes across facilities and geographical regions and measuring the national or international burden of sepsis. This is an important distinction from ASE, which was designed with particular attention to simplicity and ease of adoption across a broad range of hospitals.
Ultimately, the study by Valik and colleagues represents another important step forward in sepsis surveillance as we move further away from reliance on administrative data and towards a more objective approach using clinical data from EHRs to more reliably study changes in epidemiology and better care for sepsis patients. Further validation and comparisons of this Sepsis-3-based algorithm with ASE and other EHR-based definitions across diverse populations and EHR systems are needed to enable hospitals, policymakers and researchers to decide how best to track sepsis incidence and outcomes and tailor surveillance approaches to their particular needs.

Funding CR received support from the Agency for Healthcare Research and Quality (K08HS025008).

Competing interests None declared.

Patient consent for publication Not required.

Provenance and peer review Commissioned; internally peer reviewed.

\section{REFERENCES}

1 Rudd KE, Johnson SC, Agesa KM, et al. Global, regional, and national sepsis incidence and mortality, 1990-2017: analysis for the global burden of disease study. Lancet 2020;395:200-11.

2 Angus DC, Seymour CW, Coopersmith CM, et al. A framework for the development and interpretation of different sepsis definitions and clinical criteria. Crit Care Med 2016;44:e113-21.

3 Angus DC, Linde-Zwirble WT, Lidicker J, et al. Epidemiology of severe sepsis in the United States: analysis of incidence, outcome, and associated costs of care. Crit Care Med 2001;29:1303-10.

4 Dombrovskiy VY, Martin AA, Sunderram J, et al. Rapid increase in hospitalization and mortality rates for severe sepsis in the United States: a trend analysis from 1993 to 2003. Crit Care Med 2007;35:1244-50.

5 Martin GS, Mannino DM, Eaton S, et al. The epidemiology of sepsis in the United States from 1979 through 2000. N Engl J Med 2003;348:1546-54.

6 Rhee C, Kadri SS, Danner RL, et al. Diagnosing sepsis is subjective and highly variable: a survey of intensivists using case vignettes. Crit Care 2016;20:89.

7 Jafarzadeh SR, Thomas BS, Marschall J, et al. Quantifying the improvement in sepsis diagnosis, documentation, and coding: the marginal causal effect of year of hospitalization on sepsis diagnosis. Ann Epidemiol 2016;26:66-70.

8 Rhee C, Murphy MV, Li L, et al. Comparison of trends in sepsis incidence and coding using administrative claims versus objective clinical data. Clin Infect Dis 2015;60:88-95.

9 Rhee C, Murphy MV, Li L, et al. Improving documentation and coding for acute organ dysfunction biases estimates of changing sepsis severity and burden: a retrospective study. Crit Care 2015;19:338.

10 Gohil SK, Cao C, Phelan M, et al. Impact of policies on the rise in sepsis incidence, 2000-2010. Clin Infect Dis 2016;62:695-703.

11 Rhee C, Klompas M. Sepsis trends: increasing incidence and decreasing mortality, or changing denominator? J Thorac Dis 2020;12:S89-100. 
12 Kaukonen K-M, Bailey M, Suzuki S, et al. Mortality related to severe sepsis and septic shock among critically ill patients in Australia and New Zealand, 2000-2012. JAMA 2014;311:1308.

13 Levy MM, Gesten FC, Phillips GS, et al. Mortality changes associated with mandated public reporting for sepsis. The results of the new York state initiative. Am J Respir Crit Care Med 2018;198:1406-12.

14 Epstein L, Dantes R, Magill S, et al. Varying Estimates of Sepsis Mortality Using Death Certificates and Administrative Codes--United States, 1999-2014. MMWR Morb Mortal Wkly Rep 2016;65:342-5.

15 Ong P, Gambatese M, Begier E, et al. Effect of cause-of-death training on agreement between hospital discharge diagnoses and cause of death reported, inpatient hospital deaths, New York City, 2008-2010. Prev Chronic Dis 2015;12:E04.

16 Rhee C, Dantes RB, Epstein L, et al. Using objective clinical data to track progress on preventing and treating sepsis: CDC's new 'Adult Sepsis Event' surveillance strategy. BMJ Qual Saf 2019;28:305-9.

17 Centers for Disease Control and Prevention. Hospital toolkit for adult sepsis surveillance. Available: https://www.cdc.gov/ sepsis/pdfs/Sepsis-Surveillance-Toolkit-Mar-2018_508.pdf [Accessed 23 Mar 2018].

18 Singer M, Deutschman CS, Seymour CW, et al. The third International consensus definitions for sepsis and septic shock (Sepsis-3). JAMA 2016;315:801-10.
19 Rhee C, Dantes R, Epstein L, et al. Incidence and trends of sepsis in US hospitals using clinical vs claims data, 2009-2014. JAMA 2017;318:1241-9.

20 Valik JK, Ward L, Tanushi H, et al. Validation of automated sepsis surveillance based on the Sepsis-3 clinical criteria against physician record review in a general Hospital population: observational study using electronic health records data. BMJ Qual Saf 2020;29:735-45.

21 Seymour CW, Liu VX, Iwashyna TJ, et al. Assessment of clinical criteria for sepsis: for the third International consensus definitions for sepsis and septic shock (Sepsis-3). JAMA 2016;315:762-74.

22 Goldman LE, Chu PW, Osmond D, et al. The accuracy of present-on-admission reporting in administrative data. Health Serv Res 2011;46:1946-62.

23 Rhee C, Zhang Z, Kadri SS, et al. Sepsis surveillance using adult sepsis events simplified eSOFA criteria versus Sepsis-3 sequential organ failure assessment criteria. Crit Care Med 2019;47:307-14.

24 Seymour CW, Coopersmith CM, Deutschman CS, et al. Application of a framework to assess the usefulness of alternative sepsis criteria. Crit Care Med 2016;44:e122-30.

25 Klein Klouwenberg PMC, Ong DSY, Bonten MJM, et al. Classification of sepsis, severe sepsis and septic shock: the impact of minor variations in data capture and definition of SIRS criteria. Intensive Care Med 2012;38:811-9. 